
\title{
25 Research Soure \\ Expression and Correlation of Circulating miRNAs in Healthy Obese Children
}

\section{Feifei Ma}

Capital Institute of Pediatrics

\section{Dingding Cao}

Capital Institute of Pediatrics

\section{Zhuo Liu}

Capital Institute of Pediatrics

\section{Yuanyuan Li}

Capital Institute of Pediatrics

\section{Shengrong Ouyang}

Capital Institute of Pediatrics

Jianxin WU ( $\square$ jianxinwu_2008@126.com )

Beijing Tongren Hospital https://orcid.org/0000-0002-2335-2557

\section{Research}

Keywords: Child, Simple obesity, microRNA, target gene, target gene

Posted Date: March 18th, 2021

DOl: https://doi.org/10.21203/rs.3.rs-312584/v1

License: (c) (i) This work is licensed under a Creative Commons Attribution 4.0 International License. Read Full License 


\section{Abstract}

Objective: Obesity is an important risk factor of metabolic diseases. It is caused by the interaction of genetic, epigenetic, and environmental factors. This study aimed to identify specific circulating miRNAs for evaluating obesity in children.

Methods: Thirty children including 15 obese and 15 extremely thin were selected. The miRNA microarray was used to detect the expression of miRNAs in circulating plasma. The reliability of differential miRNA expression was verified using TaqMan qPCR. The correlation between miRNA and obesity was analyzed using multiple linear regression. Target genes for selected miRNAs were analyzed using informatics tools, and a functional network map was constructed.

Results: Thirty-six differential expression miRNAs were screened by gene chip, and seven up-regulated miRNAs were verified by TaqMan qPCR, including hsa-miR-126-3p, hsa-miR-15b-5p, hsa-miR-199a-3p, hsa-miR-20a-5p, hsa-miR-223-3p, hsa-miR-23a-3p, and hsa-miR-24-3p. Six miRNAs had significant statistical difference except hsa-miR-23a-3p. Multiple linear regression analysis showed that hsa-miR15b-5p and hsa-miR-223-3p were associated with obesity [1.649 (4.974-16.084), -1.175 (-17.852 -2.657)]. After adjusting for age and gender, these two miRNAs were still associated with obesity [1.400 (3.572-14.301), -0.973 (-15.634 -1.303)]. Among them, hsa-miR-15b-5p and hsa-miR-223 had more predicted obesity-related target genes than others. In particular, hsa-miR-15b-5p had numerous target genes associated with the FoxO, insulin, Ras, and AMPK signaling pathways.

Conclusion: The miRNA expression profile in the body circulation of obese children differs from normal children. This result is attributed to the abnormal metabolism of obese children. hsa-miR-15b-5p and hsamiR-223-3p could serve as a molecular marker for screening obese children and susceptible population of metabolic syndrome.

\section{Introduction}

The incidence rate of obesity has rapidly increased worldwide. In specific, overweight and obesity have become prevalent among children and adolescents $[1,2]$. With the rapid development of China's social economy, the continuous improvement of people's living standards, and the significant changes in family lifestyle, obese children have increasingly become a serious concern. The third national epidemiological survey on childhood obesity conducted in 2006 showed that the total detection rates of obesity and overweight among children aged $0-6$ years in China in the past 10 years were $7.2 \%$ and $19.8 \%$, respectively [3]. In recent years, the number of school-age children and adolescents with obesity and overweight have grown at a worrying speed, and the incidence rates of obesity among urban and rural children are increasing. The number of overweight and obese children in China has reached 12 million, accounting for one-thirteenth of the "overweight children" in the world. This result shows that the obesity of adolescents in China has entered a rapid epidemic period, which seriously threatens the health and life of Chinese people, and has become a major public health problem in China. 
With the rapid development of life science and technology in recent years, the explanation of development and disease mechanism has improved. MicroRNA (miRNA) is an endogenous non-coding single stranded small RNA with a length of about $22 \mathrm{nt}$. Incomplete complementary pairing with a target mRNA can degrade the target mRNA or inhibit its translation. MiRNAs are involved in almost all life activities and the pathogenesis of most diseases [4]. This study aims to analyze the circulating miRNA levels in children with simple obesity and normal weight. This study aims to explore the possibility of miRNAs as molecular markers of obesity-related metabolic abnormalities in Chinese children.

\section{Objects And Methods Research object}

For the circulating miRNA study, 30 children ( 15 boys and 15 girls) aged $8-15$ years without any intervention who underwent routine health physical examination in primary and secondary schools in Beijing were recruited from August 2015 to April 2017. The body fat index (BMI) of boy and girl students in urban and rural areas consulted by "Investigation report on Chinese students' physique and health in 2000 " was used to standardize the BMI data [5]. BMl=weight $/$ height ${ }^{2}\left(\mathrm{~kg} / \mathrm{m}^{2}\right)$ and fat mass percentage (FMP) were determined using the bioelectrical impedance method. The participants were divided into two subsets: obesity group (simple obesity refers to $B M l \geq 32 \mathrm{~kg} / \mathrm{m}^{2}$ ) and extremely thin group (lean, $\mathrm{BMI} \leq 15 \mathrm{~kg} / \mathrm{m}^{2}$ ). Participants with secondary obesity, autoimmune diseases, familial genetic diseases, hematologic diseases, various malignant tumors and recent severe infection, major trauma, or surgery within 3 months of enrollment were excluded.

\section{Sample collection}

A total of $2 \mathrm{~mL}$ venous blood samples were separately collected. The collection tubes were anticoagulated with EDTA. Blood samples were centrifuged at $2000 \mathrm{rpm} / \mathrm{min}$ for $5 \mathrm{~min}$. Plasma and red blood cells were separated and stored at $-80^{\circ} \mathrm{C}$ in a refrigerator. Plasma were used for microarray screening (miRNA screening), and the remaining blood samples were used for PCR validation.

\section{miRNA microarray assay}

Total RNA was extracted and purified using mirVanaTM PARISTM (Cat\#AM1556, Ambion, Austin, TX, US) in accordance with the manufacturer's instructions and checked for the RIN number to inspect RNA integration with an Agilent Bioanalyzer 2100 (Agilent technologies, Santa Clara, CA, US). The RNA samples were further analyzed at Shanghai Biotechnology Corporation (Shanghai, China). The expression of miRNAs, including 2006 mature human miRNAs, was profiled through miRNA microarray analysis using Agilent Human miRNA Array V19.0 (Agilent Technologies, Santa Clara, CA, USA). Differentially expressed miRNAs were identified using paired t-test with the cutoff criteria of $P<0.05$. 


\section{Quantitative polymerase chain reaction (qPCR)}

qPCR was performed using an ABI $7900 \mathrm{HT}$ sequence detection system (Applied Biosystems; Thermo Fisher Scientific, Inc.). Plasma has-miR-126-3p, has-miR-15b-5p, has-miR-199a-3p, has-miR-20a-5p, hasmiR-223-3p, has-miR-24-3p, and has-miR-23a-3p were detected by TaqMan microRNA reverse transcription kit ( $A B I, U S A)$. Given its relatively stable expression level, has-miR-1228-3p was selected as the internal standard gene [6]. The sequence of probe sequence was as follows: hsa-miR-24-3p, UGGCUCAGUUCAGCAGGAACAG; hsa-miR-223-3p, UGUCAGUUUGUCAAAUACCCCA; hsa-miR-20a-5p, UAAAGUGCUUAUAGUGCAGGUAG; hsa-miR-199a-3a-3p, ACAGUAGUCUGCACAUUGGUUA; hsa-miR-15b-5p, UAGCAGCACAUCAUGGUUUACA; hsa-miR-126-3p, UCGUACCGUGAGUAAUAAUGCG; hsa-miR-1228-3p, UCACACCUGCCUCGCCCCCC. The thermocycling conditions for quantitative PCR were $50{ }^{\circ} \mathrm{C}$ for $60 \mathrm{~s}, 95$ ${ }^{\circ} \mathrm{C}$ for $10 \mathrm{~min}, 95^{\circ} \mathrm{C}$ for $15 \mathrm{~s}$, and $60^{\circ} \mathrm{C}$ for $60 \mathrm{sec}(40 \mathrm{cycles})$. The data were processed using the relative quantification method. The relative values were measured using the $2^{-\triangle \Delta C T}$ (Livak) method.

\section{Identification of predicted miRNA target genes and functional analysis}

The Mirwalk (http://mirwalk.umm.uni-heidelberg.de/) algorithm was used to predict the target genes of the differentially expressed miRNAs. Mirwalk is an early software for predicting miRNA target genes. In Nature Methods, the first version was released in 2011, followed by the V2 version in 2015, and then the V3 version at present [7]. The 1968 predicted target genes were analyzed using Database for Annotation, Visualization, and Integrated Discovery software. The results were visualized with the functional network map plug-in for Cytoscape, with which functional enrichment was visualized and compared.

\section{Data analysis and statistics}

The microarray expression data were standardized using the internal reference gene (has-miR-1228-3p) and transformed into log 2 for subsequent analysis. The data are presented as mean \pm SD in accordance with normal distribution. Independent sample t-test was used for comparison between the two groups. The correlation between clinical features and serum miRNAs was analyzed using Pearson correlation coefficient. Multiple linear regression was used for correlation analysis between miRNA expression and obesity risk. Data were analyzed using the statistical software package SPSS 26.0 (SPSS, Inc., Chicago, IL, USA). Statistical significance was considered at $P$ value $<0.05$.

\section{Results}

\section{Participant characteristics}


The age, gender, BMI, and FMP of the two groups were statistically analyzed. No significant difference in age and gender was found between the two groups, but they significantly differed in BMI and FMP (Table 1).

Table 1

Characteristics of the participants

\begin{tabular}{|llll|}
\hline & Lean $(\mathbf{N}=13)$ & Obese $(\mathbf{N}=14)$ & $p^{\mathrm{a}}$ \\
\hline Age (year) & $10.39 \pm 0.96$ & $12.15 \pm 2.6$ & 0.029 \\
\hline Gender (male/female) & $7 / 6$ & $7 / 7$ & 0.842 \\
\hline BMI $\left(\mathrm{kg} / \mathrm{m}^{2}\right)$ & $14.24 \pm 0.62$ & $38.94 \pm 5.09$ & $<0.001$ \\
\hline FMP $(\%)$ & $9.42 \pm 1.69$ & $48.99 \pm 2.71$ & $<0.001$ \\
\hline Data are presented as the mean \pm SD. & & \\
\hline Abbreviations: BMl, body mass index; FMP, fat mass percentage; ${ }^{\text {a }}$, Student t-test. \\
\hline
\end{tabular}

\section{MiRNA expression profiling in obesity}

Through the analysis of differentially expressed genes, 34 miRNAs with significant difference $(P<0.05$, fold change $\geq 2$ times, and mean=3) were selected. After further screening by internal reference, 36 miRNAs with significant difference in expression ( $P<0.05$, fold change $\geq 2$, and mean $=3$ ) were selected. Ten differentially expressed miRNAs were identified as the research objects after the intersection analysis (Table 2). Seven up-regulated miRNAs were selected for further verification. 
Table 2

Differentially expressed miRNAs modified by the original difference and internal reference

\begin{tabular}{|llll|}
\hline Names & Pvalue & Fold change & Regulation \\
\hline hsa-miR-21-5p & $2.54 \mathrm{E}-08$ & 6.7 & down \\
\hline hsa-miR-27a-3p & $4.83 \mathrm{E}-05$ & 8.0 & down \\
\hline hsa-miR-130a-3p & $2.34 \mathrm{E}-07$ & 5.7 & down \\
\hline hsa-miR-126-3p & $4.13 \mathrm{E}-08$ & 8.3 & up \\
\hline hsa-miR-15b-5p & $2.32 \mathrm{E}-08$ & 8.9 & up \\
\hline hsa-miR-199a-3p & $3.44 \mathrm{E}-06$ & 13.2 & up \\
\hline hsa-miR-20a-5p & 0.000158 & 7.3 & up \\
\hline hsa-miR-223-3p & $2.35 \mathrm{E}-07$ & 8.4 & up \\
\hline hsa-miR-23a-3p & $1.57 \mathrm{E}-08$ & 8.1 & up \\
\hline hsa-miR-24-3p & $2.36 \mathrm{E}-07$ & 7.1 & up \\
\hline Note: Top 10 differential expression miRNAs compared with 2 groups. Lean $(\mathrm{N})=13$, Obesity $(\mathrm{N})=14$. \\
\hline
\end{tabular}

\section{Level of differential expression of miRNAs}

The reliability of microarray results was verified as follows. The level of differential miRNA expression was detected using TaqMan probe. Results showed that hsa-miR-126-3p, hsa-miR-15b-5p, hsa-miR-199a$3 p$, hsa-miR-20a-5p, hsa-miR-223-3p, and hsa-miR-24-3p were significantly different between the obese and lean groups. No significant difference in hsa-miR-23a-3p was found between the two groups (Figure 1).

\section{Correlation analysis of miRNAs expression level}

The correlation of BMI, FMP, and age with hsa-miR-126-3p, hsa-miR-15b-5p, hsa-miR-199a-3p, hsa-miR20a-5p, hsa-miR-223-3p, hsa-miR-23a-3p, and hsa-miR-24-3p was analyzed (Table 3). Results showed that the expression levels of hsa-miR-126-3p, hsa-miR-15b-5p, hsa-miR-199a-3p, hsa-miR-20a-5p, and hsa-miR-24-3p were positively correlated with BMI and FMP but not with age. No correlation was found between hsa-mir-223-3p and BMI, FMP, and age. 
Table 3

Correlation analysis of miRNA and obesity index (BMl, FMP and Age)

\begin{tabular}{|c|c|c|c|c|c|c|c|}
\hline & & $\begin{array}{l}\text { hsa-miR- } \\
126-3 p\end{array}$ & $\begin{array}{l}\text { hsa-miR- } \\
15 b-5 p\end{array}$ & $\begin{array}{l}\text { hsa-miR- } \\
\text { 199a-3p }\end{array}$ & $\begin{array}{l}\text { hsa-miR- } \\
20 a-5 p\end{array}$ & $\begin{array}{l}\text { hsa-miR- } \\
\text { 223-3p }\end{array}$ & $\begin{array}{l}\text { hsa-miR- } \\
24-3 p\end{array}$ \\
\hline \multirow[t]{2}{*}{ Age } & $r$ & 0.052 & 0.193 & 0.144 & 0.146 & 0.109 & 0.083 \\
\hline & $p$ & 0.797 & 0.336 & 0.475 & 0.468 & 0.589 & 0.681 \\
\hline \multirow[t]{2}{*}{ BMI } & $r$ & $.493^{* *}$ & $.550^{\star *}$ & $.520^{\star \star}$ & $.506^{\star *}$ & 0.370 & $.520^{* *}$ \\
\hline & $p$ & 0.009 & 0.003 & 0.005 & 0.007 & 0.058 & 0.005 \\
\hline \multirow[t]{2}{*}{ FMP } & $r$ & $.534^{\star *}$ & $.518^{\star \star}$ & $.515^{\star \star}$ & $.472^{*}$ & 0.348 & $.534^{\star *}$ \\
\hline & $p$ & 0.004 & 0.006 & 0.006 & 0.013 & 0.075 & 0.004 \\
\hline
\end{tabular}

\section{Association between miRNA and obesity risk}

The correlation between miRNA and obesity was analyzed using multiple linear regression. The increased expression levels of hsa-miR-15b-5p and hsa-miR-223-3p were related to obesity. After adjusting the sex and age of confounding factors, the above two miRNAs were still associated with obesity, as shown in Table 4.

Table 4

Multiple linear regression analysis on the relationship between miRNA and obese children

\begin{tabular}{|c|c|c|c|c|c|c|}
\hline \multirow[t]{2}{*}{ miRNA } & \multicolumn{3}{|c|}{ Model 1} & \multicolumn{3}{|c|}{ Model 2} \\
\hline & $\beta$ & $95 \% \mathrm{Cl}$ & $P$ value ${ }^{*}$ & $\beta$ & $95 \% \mathrm{Cl}$ & $P$ value* \\
\hline hsa-miR-15b-5p & 1.649 & $4.974 \rrbracket 16.084$ & 0.001 & 1.400 & $3.572 \rrbracket 14.301$ & 0.002 \\
\hline hsa-miR-223-3p & -1.175 & $-17.852 \rrbracket-2.657$ & 0.010 & -0.973 & $-15.634 \rrbracket-1.303$ & 0.022 \\
\hline \multicolumn{7}{|c|}{$\begin{array}{l}\text { Note: Independent variables of model } 1 \text { were miRNAs hsa-miR-15b-5p and hsa-miR-223-3p; } \\
\text { independent variables of model } 2 \text { were miRNAs hsa-miR- } 15 b-5 p \text { and hsa-miR-223-3p and adjustment } \\
\text { factors age and gender. It still had significance after multiple tests after correction; }{ }^{*} P<0.05 \text {. }\end{array}$} \\
\hline
\end{tabular}

\section{Pathway analysis for predicted targets of miRNAs}

Potential target genes of hsa-miR-15b-5p and hsa-miR-223-3p were predicted using the miRWalk database. Therefore, we identified 1968 predicted target genes of the two selected miRNAs. Between the two selected miRNAs, hsa-miR-15b-5p had numerous target genes in the obesity-related functions 
identified. hsa-miR-15b-5p target genes were involved in the FoxO signaling pathway, the insulin signaling pathway, the Ras signaling pathway, the AMPK signaling pathway, endocrine and other factor-regulated calcium reabsorption, the cAMP signaling pathway, the prolactin signaling pathway, signaling pathways regulating pluripotency of stem cells, the sphingolipid signaling pathway, and the thyroid hormone signaling pathway.

Figure 2 shows the pathway network for the predicted targets of the two miRNAs, which indicates the association of the various pathways of obesity, including the FoxO, insulin, Ras, and AMPK signaling pathways. Each node indicates a gene group, where a diamond node represents a miRNA and a circular node represents an mRNA. The node color represents fold changes in miRNA expression (i.e., yellow indicates upregulation).

\section{Discussion}

An increase in obesity is positively correlated with metabolic diseases, such as type 2 diabetes mellitus, dyslipidemia, atherosclerosis, hypertension, and diabetes. Epidemiological survey shows that about $35 \%$ of adults and $20 \%$ of children in the United States and other countries have developed obesity in the past 30 years [8]. The risk factors of hypertension, coronary heart disease, diabetes, metabolic syndrome, and other complications caused by obesity are independent risk factors of coronary heart disease and ischemic stroke. Weight control is a key to the prevention and treatment of chronic diseases.

In recent years, considerable evidence on miRNA studies has shown that miRNA is an important factor in the epigenetic regulation of adipogenesis $[9,10]$ and obesity $[11,12]$. A previous study $[13]$ reported that miRNAs are highly important for fat development and the whole body. Therefore, elucidating the mechanisms of miRNAs is highly important. As a new endocrine factor, circulating microRNAs can be released from various tissues and act as endocrine and paracrine messengers to promote the interaction between donor cells and tissues and recipient cells or target tissues [14]. Increasing evidence suggests that circulating miRNAs are closely related to obese adults, young subjects, and children $[15,16]$. A study of 2-6-year-old preschool obese children found that miR-190a and miR-95 in circulation can serve as molecular markers of insulin resistance in obese children [17]. In addition, a study of 70 children aged 510 years found that plasma miRNAs may be used as a potential screening tool for endothelial dysfunction in children with cardiovascular diseases [18]. Ortega [19] analyzed the response between different levels of circulating miRNA expression and obesity and weight loss in adults. Carlos found that obesity changes the miRNA profile of plasma exosomes in mice; in specific, it can increase the expression levels of miR-122, miR-192, miR-27a-3p, and miR-27b-3p. Treatment of lean mice with exosomes isolated from obese mice leads to glucose intolerance and insulin resistance [20]. In our study, the expression levels of miRNAs in circulating plasma of obese and lean children aged 8-12 years were significantly different. Among them, the expression levels of hsa-miR-126-3p, hsa-miR-15b-5p, hsa-miR-199a-3p, hsamiR-20a-5p, hsa-miR-223-3p, hsa-miR-23a-3p, and hsa-miR-24-3p were upregulated and those of hsa-miR21-5p, hsa-miR-27a-3p, and hsa-miR-130a-3p were downregulated in the obese group. The changes in miRNA expression may affect the growth and development of the body through apparent regulation, 
which plays an important role in obesity. Some studies found that circulating miR-15b-5p, miR-486-5p, and miR-122-5p are related to obesity and overweight, and analysis of four high-throughput sequencing articles revealed that these miRNAs are good candidates as obesity biomarkers [21]. In addition, miR-486, miR-146b, and miR-15b are significantly expressed in the circulation of obese children and adults with type 2 diabetes. Among them, miR-486 is involved in promoting pre-adipocyte proliferation and myotube glucose tolerance. Meanwhile, miR-146b and miR-15b are involved in the inhibition of pancreatic insulin secretion induced by high-concentration glucose and participate in the pathological process of obesity and T2D [22]. In a study, 16 of the 20 miRNAs tested in obese children are twice higher than those in the control group. Among them, miR199 and miR-122 are remarkably increased in obese children [23]. Adipogenesis is a pluripotent differentiation process in which mesenchymal stem cells can differentiate into adipocytes through adipocyte precursor cells [24]. The effects of miRNA on adipocyte differentiation are diverse. Some miRNAs promote adipocyte differentiation, and some miRNAs inhibit adipocyte differentiation [25]. MiR-143 is the first miRNA related to adipocyte differentiation. The expression of miR143 gradually increases with the differentiation and maturation of human adipose precursor cells [26]. Price found that miR-33b overexpression can inhibit pre-adipocyte proliferation and reduce lipid droplets during adipocyte differentiation [27]. In the abdominal adipose tissue of human and obese mice, high expression of miR-146a can reduce the inflammatory response of adipocytes by inhibiting the JNK and p38 signaling pathways [28]. In conclusion, the mechanism by which miRNAs regulate adipocyte differentiation remains to be explored.

The expression levels of miRNAs in circulating plasma of obese and lean children aged 8-12 years were significantly different. Among them, the expression levels of hsa-miR-126-3p, hsa-miR-15b-5p, hsa-miR199a-3p, hsa-miR-20a-5p, hsa-miR-223-3p, hsa-miR-23a-3p, and hsa-miR-24-3p were upregulated whereas those of hsa-miR-21-5p, hsa-miR-27a-3p, and hsa-miR-130a-3p were downregulated in the obese group. The changes in miRNA expression may affect the growth and development of the body through apparent regulation and play an important role in obesity. TaqMan probe were used to verify the accuracy expression of miRNA. Results showed that the expression levels of hsa-miR-126-3p, hsa-miR-15b-5p, hsamiR-199a-3p, hsa-miR-20a-5p, and hsa-miR-24-3p were positively correlated with BMI and FMP but not with age, except for hsa-miR-23a-3p. This result indicates that the above six miRNAs are markers of obesity. Multiple linear regression analysis of the correlation between miRNA and obesity showed that the expression levels of hsa-miR-15b-5p and hsa-miR-223-3p were significantly different between the obese and lean groups regardless of age and gender. However, the specific mechanism needs further study.

A pathway analysis presented the reported and predicted target genes of significantly upregulated miRNAs. hsa-miR-15b-5p and hsa-miR-223-3p were involved in many obesity-related functions, such as the FoxO, insulin, Ras, and AMPK signaling pathways. In particular, hsa-miR-15b-5p-regulated genes are involved many functions. Collectively, changes in the expression of hsa-miR-15b-5p and hsa-miR-223-3p in obese individuals may influence the pathophysiology of obesity, insulin resistance, and diabetes.

The present study attempted to identify the key miRNAs regulating obesity through microarray and correlation analyses. Results suggest that hsa-miR-15b-5p and hsa-miR-223-3p are promising targets for 
early intervention of childhood obesity. However, the sample size needs to be further expanded for further study. In addition, the related mechanisms must be elaborated at the cellular and animal levels.

\section{Conclusions}

The present study attempted to identify the key miRNAs regulating obesity through microarray and correlation analyses. Hsa-miR-15b-5p and hsa-miR-223-3p were associated with obesity, Even after adjusting for age and gender. Results suggest that hsa-miR-15b-5p and hsa-miR-223 had more predicted obesity-related target genes than other miRNAs. In particular, hsa-miR-15b-5p had numerous target genes associated with the FoxO, insulin, Ras, and AMPK signaling pathways. hsa-miR-15b-5p and hsa-miR-223$3 p$ are promising targets for early intervention of childhood obesity.

\section{Abbreviations}

Has-miR: homo sapiens-microRNA; FMP: fat mass percentage; BMI: body fat index; qPCR: quantitative polymerase chain reaction; Fox01: Forkhead box-containing protein, 0 subfamily 1; AMPK: Adenosine $5{ }^{\prime}-$ monophosphate (AMP)-activated protein kinase; cAMP: Cyclic Advenosine Monophosphato; JNK: c-Jun $\mathrm{N}$-terminal kinase.

\section{Declarations}

\section{Ethics approval and consent to participate}

The current study was approved by the Ethics Committee of Capital Institute of Pediatrics (committee's reference number: SHERLLM 2012015).

\section{Consent for publication}

Not applicable

\section{Availability of data and materials}

All data generated and analysed during the current study are available from the corresponding author on reasonable request.

\section{Competing interests}

The authors declare that they have no competing interests. 


\section{Founding}

The current study was supported by China Postdoctoral Science Foundation (grant no. 2018M631390) and Capital Institute of Pediatrics Foundation (FX-2019-04). This study was also supported, in part, by the CAMS Central Public Welfare Scientific Research Institute Basal Research Expenses (2016ZX310182-3), the CAMS Initiative for Innovative Medicine (2016-I2M-1-008) and the Special Fund of the Pediatric Medical Coordinated Development Center of Beijing Hospitals Authority (XTZD20180402).

\section{Authors' contributions}

F.M and J.W conceived and designed the study; Y.L and Z.L collected the information; F.M and D.C performed experiments $₫ S . O$ analyzed the data; F.M drafted the manuscript; J.W revised the manuscript. All authors have read and approved the final version of the manuscript.

\section{Acknowledgements}

Not applicable

\section{Author details}

${ }^{1}$ Department of Biochemistry and Immunology, Capital Institute of Pediatrics, 2 Yabao street, Beijing 100020, People's Republic of China

${ }^{2}$ Institute of Basic Medical Sciences and School of Basic Medicine, Chinese Academy of Medical Sciences and Peking Union Medical College, 5 Dongdansantiao, Beijing 100005, People's Republic of China

${ }^{3}$ Beijing TongRen Hospital, Capital Medical University, 17 Hougou Street, Chong Wen Men, Beijing 100730, People's Republic of China

\section{References}

1. Seema Kumar, Tara Kaufman. Childhood obesity. Panminerva Med. 2018; 60(4):200-212.

2. Seema Kumar, Aaron S Kelly. Review of Childhood Obesity: From Epidemiology, Etiology, and Comorbidities to Clinical Assessment and Treatment. Mayo Clin Proc. 2017; 92(2):251-265.

3. Ji C Y, Cheng T O. Epidemic increase in overweight and obesity in Chinese children from 1985 to 2005. Int J Cardiol. 2009; 132(1): 1-10.

4. Flodmark C E, Lissau I, Pietrobelli A. Child and adolescent obesity: why we need to fight!. Acta Paediatr Suppl. 2005; 94(448): 4-7. 
5. Research Group of Chinese Students Physique and Health. Report on the physical fitness and health surveillance of Chinese school students. Beijing: Higher Education Press; 2002.

6. Jian Zhou, Lei Yu, Xue Gao, Jie Hu, Jiping Wang, Zhi Dai, Jie-Fei Wang, Zhiyong Zhang, Shaohua Lu, Xiaowu Huang, Zheng Wang, Shuangjian Qiu, Xiaoying Wang, Guohuan Yang, Huichuan Sun, Zhaoyou Tang, Ying Wu, Hongguang Zhu, Jia Fan. Plasma microRNA panel to diagnose hepatitis B virus-related hepatocellular carcinoma. J Clin Oncol. 2011; 29(36):4781-8.

7. Carsten Sticht, Carolina De La Torre, Alisha Parveen, Norbert Gretz. miRWalk: An online resource for prediction of microRNA binding sites. PLoS One. 2018; 18;13(10):e0206239.

8. Flegal K M, Carroll M D, Ogden C L, et al. Prevalence and trends in obesity among US adults, 19992008. JAMA. 2010; 303(3): 235-241.

9. McGregor RA, Choi MS. MicroRNAs in the regulation of adipogenesis and obesity. Curr Mol Med. 2011; 11(4):304-16.

10. Alexander R, Lodish H, Sun L. MicroRNAs in adipogenesis and as therapeutic targets for obesity. Expert Opin Ther Targets. 2011; 15(5): 623-36.

11. Hilton C, Neville MJ, Karpe F. MicroRNAs in adipose tissue: their role in adipogenesis and obesity. Int J Obes (Lond). 2013; 37: 325-32.

12. Wang R, Hong J, Cao Y, Shi J, Gu W, Ning G, et al. Elevated circulating microRNA-122 is associated with obesity and insulin resistance in young adults. Eur J Endocrinol. 2015; 172(3):291-300.

13. Mudhasani R, Puri V, Hoover $\mathrm{K}$, et al. Dicer is required for the formation of white but not brown adipose tissue. J Cell Physiol. 2011; 226(5): 1399-1406.

14. Chenbo Ji, Xirong Guo. The clinical potential of circulating microRNAs in obesity. Nat Rev Endocrinol. 2019; 15(12):731-743.

15. Arner P, Kulyté A. MicroRNA regulatory networks in human adipose tissue and obesity. Nat Rev Endocrinol. 2015; 11(5):276-88.

16. [16] Deiuliis JA. MicroRNAs as regulators of metabolic disease: pathophysiologic significance and emerging role as biomarkers and therapeutics. Int J Obes (Lond). 2016; 40(1):88-101.

17. Masotti A, Baldassarre A, Fabrizi M, et al. Oral glucose tolerance test unravels circulating miRNAs associated with insulin resistance in obese preschoolers. Pediatr Obes. 2017; 12(3): 229-238.

18. Khalyfa A, Kheirandish-Gozal L, Bhattacharjee R, et al. Circulating microRNAs as Potential Biomarkers of Endothelial Dysfunction in Obese Children. Chest. 2016; 149(3):786-800.

19. Ortega FJ, Mercader JM, Catalán V, Moreno-Navarrete JM, Pueyo N, Sabater M, et al. Targeting the circulating microRNA signature of obesity. Clin Chem. 2013; 59(5):781-92.

20. Carlos Castaño, Susana Kalko, Anna Novials, Marcelina Párrizas. Obesity-associated exosomal miRNAs modulate glucose and lipid metabolism in mice. Proc Natl Acad Sci U S A. 2018 27; 115(48):12158-12163.

21. Carlos Andrés Rico Flórez, Herney Andrés García-Perdomo, Mildrey Mosquera Escudero. MicroRNAs associated with overweight and obesity in childhood. Microrna. 2020; 9(4):255-265. 
22. Xianwei Cui, Lianghui You, Lijun Zhu, Xing Wang, Yahui Zhou, Yun Li, Juan Wen, Yankai Xia, Xinru Wang, Chenbo Ji, Xirong Guo. Change in circulating microRNA profile of obese children indicates future risk of adult diabetes. Metabolism. 2018; 78:95-105.

23. M D Thompson, M J Cismowski, M Serpico, A Pusateri, D R Brigstock. Elevation of circulating microRNA levels in obese children compared to healthy controls. Clin Obes. 2017; 7(4):216-221.

24. Heinonen S, Saarinen L, Naukkarinen J, Rodríguez A, Frühbeck G, Hakkarainen A, et al. Adipocyte morphology and implications for metabolic derangements in acquired obesity. Int $\mathrm{J}$ Obes (Lond). 2014; 38(11):1423-31.

25. Klöting N, Berthold S, Kovacs $P$, et al. MicroRNA expression in human omental and subcutaneous adipose tissue. PLoS One. 2009; 4(3):e4699.

26. Esau C, Kang X, Peralta E, et al. MicroRNA-143 regulates adipocyte differentiation. J Biol Chem. 2004; 279(50): 52361-52365.

27. Price NL, Holtrup B, Kwei SL, et al. SREBP-1c/MicroRNA 33b Genomic Loci Control Adipocyte Differentiation. Mol Cell Biol. 2016; 1;36(7):1180-93.

28. Roos J, Enlund E, Funcke JB, et al. miR-146a-mediated suppression of the inflammatory response in human adipocytes. Sci Rep. 2016; 6:38339.

\section{Figures}



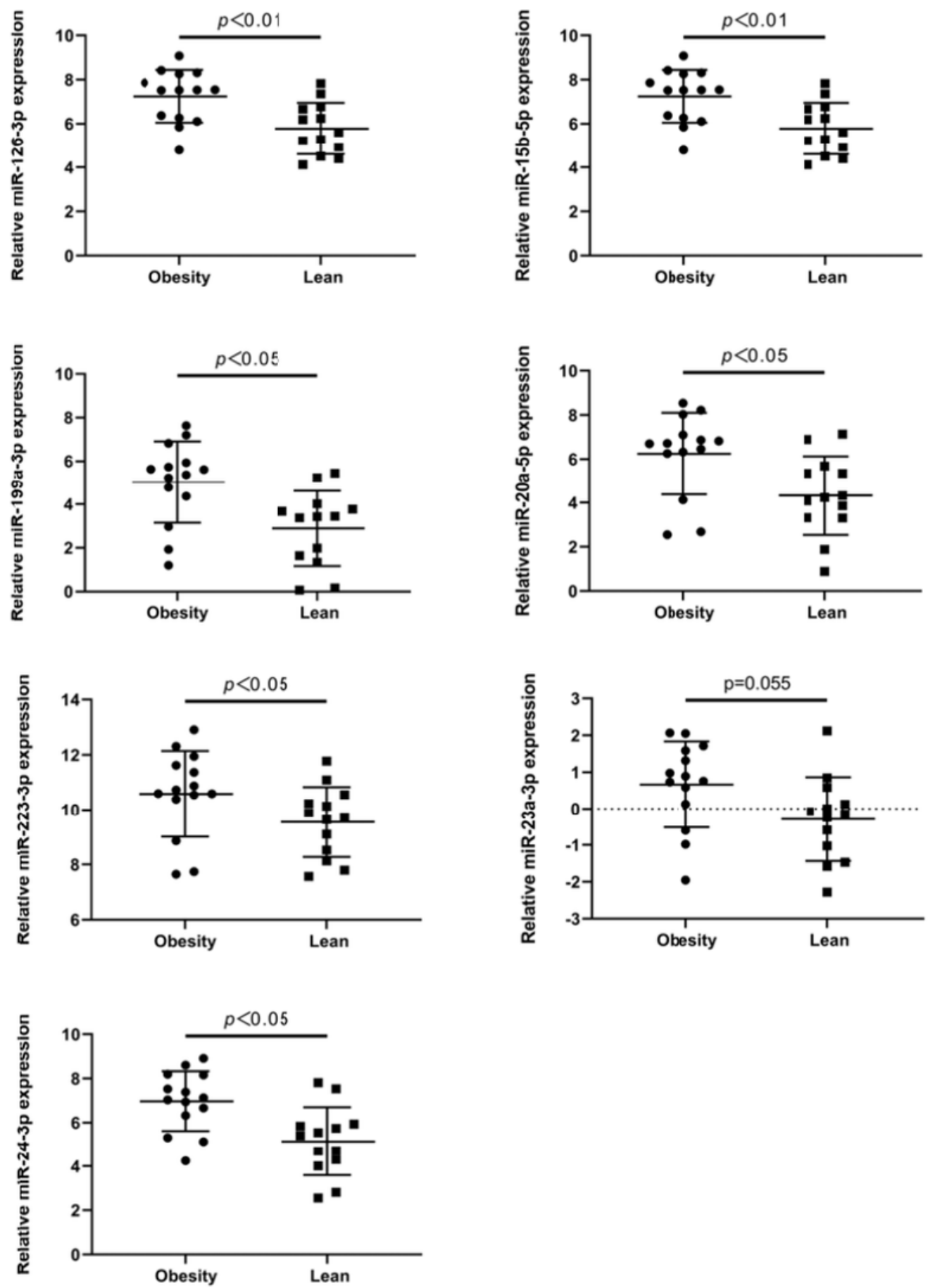

\section{Figure 1}

Expression of miRNAs detected by TaqMan probe. Comparison of the circulating miRNA expression profiles in lean and obese participants. Graphs show the mean level with the interquartile range. Onesided t-test and Wilcoxon rank test were used to determine the significant differences between groups. All error bars indicate mean \pm SEM. 


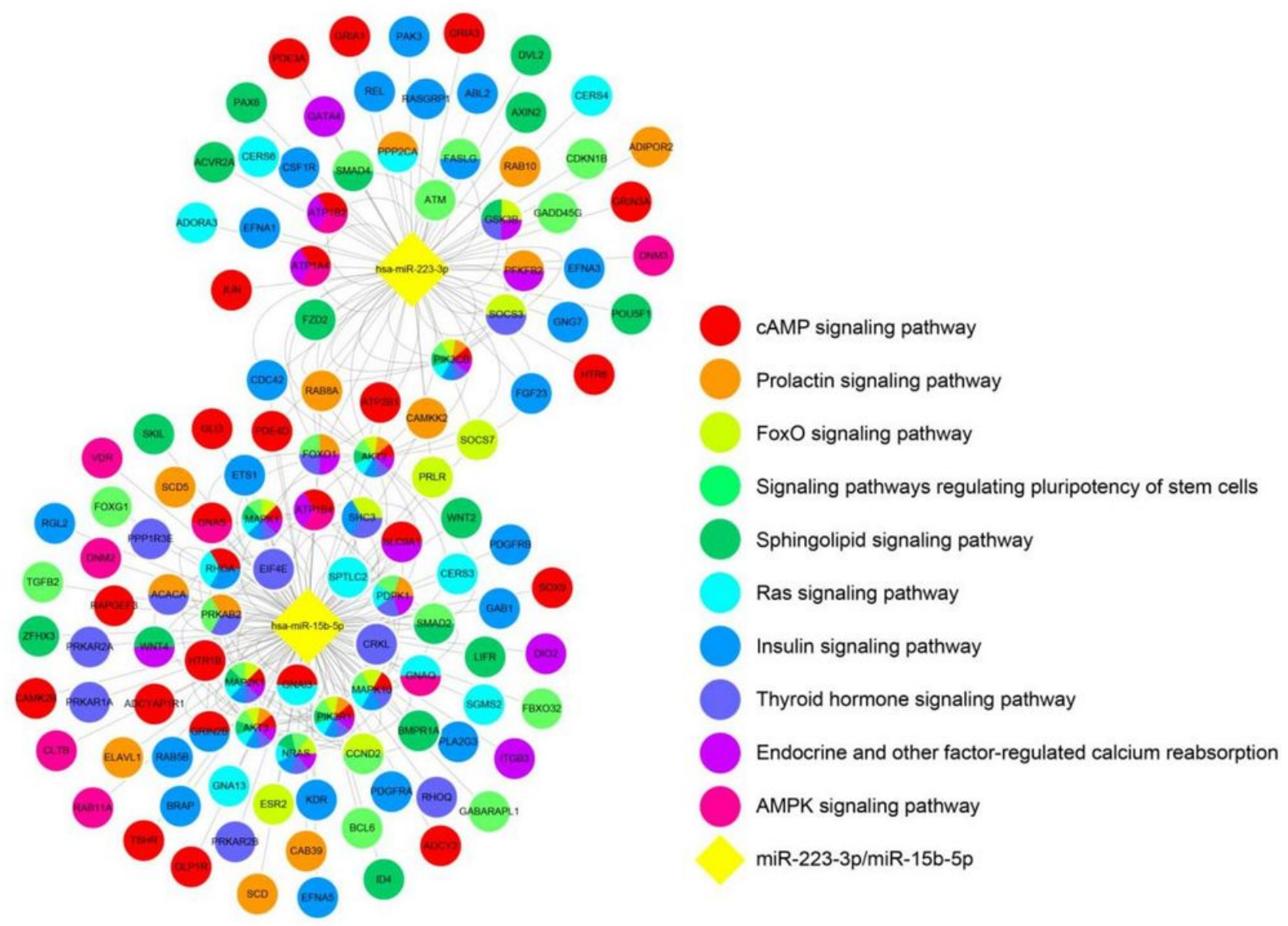

Figure 2

Mapping of target genes for miRNA-15b-5p and miR-223-3p that were significantly upregulated in obese participants. The landscape of genes mapped to biological processes was visualized using Cytoscape software. Each diamond node indicates a miRNA, and a circular node represents a target mRNA. The node color represents the pathway involved by the target genes. 